\title{
Autoeficacia en escolares adolescentes: su relación con la depresión, el rendimiento académico y las relaciones familiares
}

\author{
Iris X. Galicia-Moyeda*, Alejandra Sánchez-Velasco y Francisco J. Robles-Ojeda \\ Facultad de Estudios Superiores Iztacala. Universidad Nacional Autónoma de México
}

\begin{abstract}
Resumen: Las relaciones familiares son estimadas como elementos mediadores en diferentes tipos de autoeficacia; no obstante existe poca evidencia sobre la mediación de las relaciones familiares con la autoeficacia académica. Con respecto a ésta última se ha reportado su relación con la ansiedad y poco se ha documentado el vínculo con la depresión. En este trabajo se explora la relación entre la depresión, la autoeficacia académica, la dinámica familiar y el rendimiento académico. Participaron ochenta alumnos de educación secundaria, divididos en dos grupos en función de sus puntajes del Inventario de Depresión de Kovacs: en uno de ellos se incluyeron los que fueron identificados como deprimidos severos y en el otro, los que se diagnosticaron sin depresión. A ambos grupos les fueron aplicadas dos escalas: Clima Social en la Familia y Autoeficacia. Los resultados revelan una relación inversa entre depresión y autoeficacia total. La cohesión familiar correlaciona positivamente con la autoeficacia total y con el factor de autoeficacia académica en los sujetos sin depresión, en tanto que en los sujetos deprimidos severos las relaciones familiares conflictivas correlacionan de manera negativa tanto en el factor de autoeficacia social como en la autoeficacia académica.

Palabras clave: Adolescencia; autoeficacia; depresión; dinámica familiar; cohesión familiar; conflicto; rendimiento académico.
\end{abstract}

Title: Self-efficacy in school age adolescents: its relationship with depression, academic achievement and family relationships.

Abstract: Family relationships are regarded as mediating elements in different types of self- efficacy. However, there is few evidence on the mediating of family relationships with academic self efficacy. In respect to the latter, its relation to anxiety has been reported and its link with depression has been poorly documented. This work explores the relationship between depression, academic self-efficacy, family dynamics and academic achievement. Eighty middle school students participated, divided into two groups by their scores in Kovacs' Inventory of Depression. One group was formed with those students diagnosed with severe depression; the other one was formed with students diagnosed without depression. Both groups were applied two scales: that of Family Enviromental Scale and that of Self Efficacy. The results show an inverse relationship between depression and total self-efficacy. Family cohesion positively correlates total self efficacy and the factor of academic self efficacy on subjects without depression, while, on subjects severely depressed, conflictive family relations correlate in a negative way both the social self efficacy factor and the total academic self efficacy.

Key words: Adolescence; self-efficacy; depression; family relationships; familiy cohesion; conflict; academic achievement.

\section{Introducción}

El desempeño de los individuos se encuentra relacionado con las creencias que tienen sobre sus capacidades en una situación determinada. Dichas creencias conforman lo que se ha denominado como autoeficacia. Las creencias que las personan poseen acerca de su eficacia para controlar los eventos que afectan sus vidas, influyen en las elecciones que realizan, en sus aspiraciones, en el nivel de esfuerzo y perseverancia, en la resistencia a la adversidad, en la vulnerabilidad al estrés, en la depresión y el desempeño (Bandura, 1997). Hay que señalar que la autoeficacia es específica, es decir atiende a dominios particulares; así, se entiende la autoeficacia académica como aquella referida a ejecuciones académicas. Por lo general, sus manifestaciones se presentan, entre otros aspectos, la persistencia en actividades académicas, así como con la motivación académica (Ruiz, 2005). Otro elemento estudiado es el logro académico, el cual ha sido abordado de diversas maneras, principalmente en las destrezas cognitivas básicas, en el trabajo de un curso académico y en pruebas estandarizadas. En estos estudios se ha comprobado que el logro académico esta fuertemente relacionado con las creencias de la autoeficacia. Los estudiantes con altos logros académicos son aquellos que posen creencias positivas de sus capacidades académicas (Zimmerman, 1999).

También existe una relación positiva entre autoeficacia y rendimiento académico general, entendido éste como el

* Dirección para correspondencia [Correspondence address]: Iris X. Galicia Moyeda. Av. de los Barrios No 1 Tlalnepantla, Estado de México. E-mail: iris@servidor.unam.mx promedio acumulado de los puntos obtenidos por los estudiantes en sus estudios. Tal relación se presenta en muestras de estudiantes de diversas edades, tanto en niños como en adolescentes (Ellias y Ross, 2002; Contreras, Espinosa, Esguerra, Haikal, Polanía y Rodríguez, 2005). Diversos estudios valoran la autoeficacia de estudiantes de nivel licenciatura, encontrando una alta relación entre la autoeficacia y las notas escolares obtenidas (Ruiz, 2005); similarmente se vio una relación positiva entre el nivel de autoeficacia percibida y el número de materias aprobadas en estudiantes de licenciatura (Breso, Salanova, Martínez, Grau y Agut, 2004); por ejemplo, en un meta análisis realizado por Green (citado en Pérez y Delgado, 2006), el promedio anual de calificaciones en Lengua se asocia de manera positiva con la autoeficacia.

Hay que señalar que se ha planteado que las creencias relativas a la capacidad de manejar las demandas de las tareas influyen sobre los estados emocionales como la ansiedad y la depresión. No obstante, en el campo académico, ha sido más reportada la relación entre la autoeficacia y la ansiedad (Zimmerman, 1999 y Contreras et al., 2005) y menos documentado el vínculo de la autoeficacia académica y la depresión. Con respecto ésta última se ha identificado una relación negativa entre la depresión y el aprovechamiento académico, es decir, mientras más alto sea el nivel de depresión, menor será el rendimiento escolar (Campo-Arias et al., 2005; Galicia, Sánchez y Robles, 2009; Pérez y Urquijo, 2001). Particularmente se observa una relación más estrecha con el desempeño en materias específicas como las matemáticas y el inglés (Pérez y Urquijo, 2001 y De la Peña, Estrada, Almeida y Páez, 1999) ya que los promedios obtenidos en estas materias se correlacionan negativamente de manera significativa con los puntajes obtenidos en las pruebas de 
depresión. Dado que el rendimiento académico es un factor importante para la valoración social de los adolescentes, es por ello que se establece una relación con la depresión quizás debido a que el desempeño que tienen en la escuela los hace percibirse capaces o incapaces de actuar adecuadamente en una de las tareas importantes en su vida: sus actividades escolares. En especial, el resultado de las evaluaciones en las materias que son percibidas socialmente como las más difíciles y complejas, ya que poseen una alta carga amenazante para los alumnos de secundaria, pudiera influir en la creencia de su capacidad académica. De ahí que es factible suponer que el rendimiento académico y la percepción que el alumno tenga sobre su desempeño en general y en particular en esas asignaturas guarde alguna relación con el nivel de depresión en que se encuentre. Por lo que uno de los objetivos de este trabajo es analizar la relación que pueda darse entre la autoeficacia, la depresión y el rendimiento escolar en alumnos del nivel de secundaria.

No deja de ser importante el análisis de las relaciones familiares en el desarrollo de la autoeficacia académica. Los principales contextos de desarrollo de la autoeficacia en los inviduos son la familia, los pares y la escuela. Las interacciones surgidas en tales contextos nutren de manera importante los recursos que aparecen en la vida del individuo, contribuyendo al desarrollo de un adecuado o inadecuado sentido de auto-eficacia y permitiendo la evolución desde el control externo hasta la autorregulación personal (Pastorelli, Caprara, Barbaranelli, Rola, Rozsa y Bandura, 2001). No obstante, la investigación centrada en los antecedentes familiares del desarrollo de las creencias de autoeficacia es escasa (Schneewind, 1999; Caprara, Pastorelli, Regalia, Barbaranelli y Bandura, 2005). Existen investigaciones que se interesan en examinar las prácticas parentales que fortalecen las creencias de control interno y la subsecuente influencia en las creencias de autoeficacia. Los padres que tienden a tener hijos con una mayor orientación de control interno son aquellos que ofrecen un entorno familiar estimulante, que responden consistente y contingentemente ante la conducta de sus hijos, así como los que promueven la independencia, la autonomía y desalientan las interacciones intrusivas; usan técnicas disciplinarias más inductivas y se relacionan emocionalmente de manera reconfortante (Schneewind, 1999). Esta orientación hacia el control interno podría ser la fuente de creencias globales sobre la confianza que tiene el individuo en la capacidad para solventar las situaciones de estrés y considerarsele como una expectativa de autoeficacia generalizada (Sanjuán, Pérez-García y Bermúdez 2000; Luszcynska, Scholz y Schwarzer, 2005 y Rueda y Pérez-García, 2004).

Así pues, las relaciones familiares pueden ser estimadas como elementos mediadores en diferentes tipos de autoeficacia, por ejemplo se ha estudiado la importancia de tales relaciones en la autoeficacia romántica (Barrera y Vargas, 2005) y en la autoeficia vinculada a conductas de salud como en el manejo de diversas enfermedadades (Rueda y PérezGarcía, 2004, Avendaño y Barra, 2008). No obstante, no se han encontrado reportes que estudien la mediación de las re- laciones familiares con la autoeficacia académica en el contexto latinoamericano, de ahí que en este trabajo exista el interés de explorar tal relación. De tal suerte que este interés, aunado al expresado anteriormente, conformen el propósito general de esta investigación que consiste en el analizar la vínculo entre la autoeficacia, la depresión, el rendimiento escolar y las relaciones familiares en alumnos del nivel de secundaria.

\section{Método}

\section{Participantes}

Ochenta alumnos, cincuenta mujeres y treinta hombres, cuya edad fluctuó de los 12 a los 15 años, presentándose la moda en los 14 años. A la mitad de ellos les fue identificada depresión severa de acuerdo al Inventario de Depresión de Kovacs y el resto, sin depresión.

\section{Instrumentos}

Escala de Autoeficacia para Niños (Bandura, 1990; Pastorelli et al., 2001), en su versión y adaptación española por Carrasco y Del Barrio (2002). Esta escala está destinada para individuos de 8 a 15 años de edad. Consta de 34 reactivos cuyo formato de respuesta está graduado de cero a cinco $(1 \ll \mathrm{Fa}$ tal»; 2 «no muy bien»; 3 «bien»; 4 «muy bien»; y 5 «fenomenal»), con los cuales se pretende evaluar la percepción de eficacia que el sujeto posee en tres ámbitos: académico, social y de control. El factor de autoeficacia académica explora la capacidad percibida para dirigir el propio aprendizaje, las expectativas académicas personales, parentales y de los profesores. El segundo factor, la autoeficacia social, incluye aspectos tales como la capacidad percibida por el sujeto para las relaciones entre iguales, asertividad y actividades de ocio y tiempo libre. El último factor, la eficacia percibida de autocontrol, atiende la capacidad percibida por el sujeto para resistirse a los iguales ante la involucración en actividades de riesgo, relacionadas con la transgresión de normas.

Inventario de Depresión de Kovacs. Instrumento de escrutinio diseñado para poblaciones de entre los 8 y los 17 años de edad. Está constituido por 27 reactivos, con tres posibilidades de respuesta. La persona evaluada tiene que seleccionar sólo la que sea acorde a su estado emocional en las dos últimas semanas. Este inventario evalúa tres factores: depresión, falta de entusiasmo y relaciones interpersonales. Diagnostica tres niveles de depresión: leve (7 a 13 puntos), moderada (14 a 19 puntos) y severa (más de 20 puntos). La designación de ausencia de depresión corresponde a un puntaje de 0 a 6 puntos.

Escala de Clima Social en la Familia (Family Environment Scale, FES) elaborada por Moss, Moss y Trickett (en la adaptación española por Seisdedos, De la Cruz y Cordero, 1995). La escala esta compuesta por 90 reactivos que se responden de manera dicotómica (verdadero o falso) y permiten apreciar las características socio-ambientales de la familia. Los 
reactivos están subdivididos en 10 subescalas que se agrupan en tres dimensiones: a) Relaciones (Cohesión, Expresividad, y Conflicto), b) Desarrollo (Autonomía, Actuación, Intelectual - Cultural, Social - Recreativo y Moralidad- Religiosidad) y c) Estabilidad (Organización y Control).

\section{Procedimiento}

El inventario de Kovacs se aplicó a doscientos cincuenta y siete alumnos de educación secundaria. Los alumnos participaron de manera voluntaria como parte de las actividades propuestas en la asignatura Orientación Educativa y Vocacional para la elaboración de su expediente en dicha asignatura. Los resultados fueron informados de manera personal a los alumnos así como a sus padres, en los casos necesarios fueron derivados a un servicio especializado.

A partir de los puntajes obtenidos, se eligieron aquellos participantes que tuvieran puntajes extremos, quedando cuarenta alumnos con tendencia depresiva severa y cuarenta diagnosticados como normales. A los ochenta alumnos se les solicitó su colaboración en el estudio, una vez obtenida su aceptación, se les aplicaron la Escala de Clima Social de la Familia (FES) y la Escala de Autoeficacia. Se formaron grupos de veinte estudiantes a quienes se reunió en un salón de usos múltiples para la aplicación de ambos instrumentos durante dos sesiones, una para cada instrumento. Una vez obtenido sus resultados se les proporcionó información sobre ellos con la colaboración del servicio de orientación de la escuela. Aunado a esto, se obtuvieron sus calificaciones mediante la consulta autorizada del archivo de control escolar de la institución educativa a la que pertenecían.

\section{Resultados}

\section{Descripción general de la muestra}

Las mujeres presentaron mayores puntajes de depresión que los hombres, siendo tal diferencia significativa tanto para los puntajes totales del instrumento como para los factores de relaciones interpersonales y depresión (ver Tabla 1)

Tabla 1. Muestra el promedio de los puntajes de derivados del Inventario de Depresión de Kovacs en función del género. También se presentan los valores de $t$ y del nivel de significancia obtenidos para cada factor y el total de dicho inventario.

\begin{tabular}{llcccc}
\hline & Inventario de Depresión & Mujeres & Hombres & $t$ & $p$ \\
\hline \multirow{2}{*}{ Factores } & Puntaje Total & 17.16 & 11.31 & 2.360 .021 \\
de & Falta de entusiasmo & 4.31 & 3.19 & 1.523 .132 \\
Depresión & Depresión & 7.75 & 4.63 & 2.765 .007 \\
Relaciones Interpersonales & 5.13 & 3.50 & 2.016 .047 \\
\hline
\end{tabular}

Con respecto al rendimiento académico las mujeres presentaron un promedio de calificaciones mayor en relación al de los hombres cuya dicha diferencia fue significativa al nivel de .005 , de manera similar las diferencias en calificaciones para cada materia también fueron significativas, presentándose los mejores puntajes en las mujeres. No se encontraron diferencias significativas en cuanto a género en los puntajes de la escala de autoeficacia y sus factores y tampoco en la escala de clima familiar y sus subescalas.

\section{Depresión y Rendimiento Académico.}

Los alumnos clasificados como depresivos severos obtuvieron calificaciones menores que los sujetos considerados normales. Las diferencias se presentan tanto en el promedio general así como en las calificaciones obtenidas en cada materia. Al aplicar la prueba $t$ de Student se advierte que tales diferencias son significativas en todos los casos excepto en una sola materia, Educación Física.

\section{Depresión y Dinámica Familiar}

Con objeto de corroborar algunas relaciones encontradas en estudios anteriores, se realizaron análsis de diferencias de medias del clima social de la familia entre los grupos de adolescentes deprimidos severos y no deprimidos, así como las correlaciones entre los puntajes obtenidos en el Kovacs y en la escala FES. El análisis de diferencias de medias reveló que las puntuaciones obtenidas por el instrumento de clima familiar son diferentes entre los alumnos identificados como depresivos severos y los sujetos no deprimidos. Estos últimos tienen mayores puntajes en todas las subescalas de dicho instrumento, a excepción de la subescala de conflicto. Todas las diferencias resultan significativas a excepción de las presentadas en las subescalas que valoran el desarrollo moral y de la autonomía, así como el control (ver Tabla 2). Lo anterior indica que tanto los sujetos deprimidos severos como los no deprimidos no se distinguen por el tipo de control que se establece en sus familias ni por las posibilidades que se les proporcione de desarrollar su autonomía y el aspecto moral y religioso.

Tabla 2. Se presentan las medias obtenidas por los sujetos normales y deprimidos severos en cada una de las subescalas de las tres dimensiones de la escala de Clima Social en la Familia. Así mismo se muestran los valores de la $t$ de Student y el nivel de significación obtenido.

\begin{tabular}{llllcc}
\hline Dimensiones & Subescalas & Normales & Severos & $t$ & $p$ \\
\hline Relaciones & Cohesión & 52.93 & 42.15 & 5.372 & .000 \\
& Expresividad & 50.68 & 44.85 & 3.125 & .002 \\
& Conflicto & 47.20 & 55.65 & -5.412 & .000 \\
\multirow{5}{*}{ Desarrollo } & Autonomía & 46.78 & 43.08 & 1.725 & .088 \\
& Actuación & 51.33 & 47.80 & 2.031 & .046 \\
& Intelectual-Cultural & 52.73 & 48.78 & 2.381 & .020 \\
& Social-Recreativo & 53.38 & 49.20 & 3.040 & .003 \\
\multirow{5}{*}{ Estabilidad } & Moral-Religioso & 57.63 & 54.25 & 1.823 & .072 \\
& Organización & 52.53 & 46.95 & 2.985 & .004 \\
& Control & 54.08 & 52.45 & 1.000 & .320 \\
\hline
\end{tabular}

En la Tabla 3 se muestran las correlaciones entre los diversos factores de los instrumentos de clima social de la familia y de depresión. Como dato general se observan correlaciones negativas, moderadas-bajas y significativas en las tres subescalas (cohesión, expresividad y conflicto) que conforman la dimensión de Relaciones y los factores del instrumento de depresión. En la dimensión de Desarrollo sólo dos escalas exhiben una correlación negativa y significativa con 
la depresión, no obstante esa correlación es baja. Dichas escalas son la intelectual-cultural y la social- recreativa. En la dimensión de estabilidad la subescala de organización presenta correlaciones negativas significativas pero con niveles bajos y la subescala de control no reporta correlaciones sig- nificativas con la depresión. De lo anterior se desprende que es factible que las subescalas que constituyen la dimensión de Relaciones sean las que distinguen a los sujetos deprimidos severos de los no deprimidos.

Tabla 3. Correlaciones entre los puntajes totales y de los tres factores del Inventario de Depresión de Kovacs y los puntajes de cada una de las subescalas de la Escala de Clima Social en la Familia.

\begin{tabular}{|c|c|c|c|c|c|}
\hline \multicolumn{2}{|c|}{ Clima Social en la Familia } & \multicolumn{4}{|c|}{ Inventario de Depresión de Kovacs } \\
\hline Dimensiones & Subescalas & Total & Falta de entusiasmo & Depresión & Relaciones Interpersonales \\
\hline \multirow[t]{3}{*}{ Relaciones } & Cohesión & $-.526(* *)$ & $-.448(* *)$ & $-.504(* *)$ & $-.503(* *)$ \\
\hline & Expresividad & $-.406(* *)$ & $-.337(* *)$ & $-.432(* *)$ & $-.331(* *)$ \\
\hline & Conflicto & $.492(* *)$ & $.450(* *)$ & $.481(* *)$ & $.429(* *)$ \\
\hline \multirow[t]{5}{*}{ Desarrollo } & Autonomía & -.208 & -.195 & $-.223(*)$ & -.148 \\
\hline & Actuación & -.207 & -.149 & $-.228(*)$ & -.187 \\
\hline & Intelectual-Cultural & $-.287(* *)$ & -.128 & $-.321(* *)$ & $-.315(* *)$ \\
\hline & Social-Recreativo & $-.340(* *)$ & $-.289(* *)$ & $-.312(* *)$ & $-.342(* *)$ \\
\hline & Moral-Religioso & -.201 & -.181 & -.181 & -.200 \\
\hline \multirow[t]{2}{*}{ Estabilidad } & Organización & $-.284(* *)$ & $-.266(*)$ & $-.263(*)$ & $-.263(*)$ \\
\hline & Control & -.114 & -.072 & -.100 & -.145 \\
\hline
\end{tabular}

$* p<.05 ; * * p<.001$

Se realizó un análisis discriminante con las puntuaciones de subescalas del instrumento de clima social en la familia, el cual muestra una sola función discriminante (autovalor= .693 que explica el $100 \%$ de las diferencias existentes entre grupos, correlación canónica de .640 y lambda moderada .591 y significativa .001). Las variables que, de acuerdo a las correlaciones con funciones discriminantes, distinguen a los dos grupos de deprimidos son las relativas a las relaciones: conflicto y cohesión (-.736 y .731, respectivamente), en tanto las que menos contribuyen son las de autonomía (.235) y control (.136), información que confirma la obtenida por los análisis anteriores.

\section{Depresión y Autoeficacia}

Existe una relación inversa entre depresión y autoeficacia. Los sujetos que obtuvieron niveles bajos de depresión y que fueron clasificados como no deprimidos, presentan pun- tajes de autoeficacia más altos que los identificados como deprimidos severos $(t=3.533, p=.001)$. Las puntuaciones obtenidas en los factores que constituyen la escala de autoeficacia también presentan dicha relación; las diferencias en la autoeficacia debidas al nivel de depresión sólo fueron significativas para la autoeficacia social $(t=3.165, p=.002) \mathrm{y}$ académica $(t=3.636, p=.000)$. La autoeficacia de control parece manifestarse de manera similar tanto en sujetos deprimidos severos y en sujetos no deprimidos $(t=1.244, p=$ .217).

Un análisis de correlación también revela una relación inversa entre depresión y autoeficacia. En la Tabla 4 se pueden observar índices bajos, negativos y significativos entre los factores de depresión evaluados en el inventario de Kovacs y únicamente en los factores de autoeficacia académica y la social de la escala de autoeficacia. Las correlaciones de la autoeficacia de control y los puntajes de los factores del Kovacs fueron negativos, aún más bajos y no significativos.

Tabla 4. Muestra las correlaciones entre los diversos puntajes de la escala de Autoeficacia Académica y del Inventario de Depresión de Kovavs. Autoeficacia Percibida Inventario de Depresión de Kovacs

\begin{tabular}{|c|c|c|c|c|}
\hline & Total & Falta de entusiasmo & Depresión & Relaciones Interpersonales \\
\hline Total & $-.303(* *)$ & $-.323(* *)$ & $-.254(*)$ & $-.286(* *)$ \\
\hline Autoeficacia académica & $-.296(* *)$ & $-.348(* *)$ & -.207 & $-.308(* *)$ \\
\hline Autoeficacia social & $-.299(* *)$ & $-.262(*)$ & $-.302(* *)$ & $-.261\left(^{*}\right)$ \\
\hline Autoeficacia de control & -.121 & -.120 & -.139 & -.073 \\
\hline
\end{tabular}
$* p<.05 ; * * p<.001$

$\mathrm{El}$ análisis discriminante efectuado revela una correlación canónica baja (.415) con un índice de lambda alto (.827) y un nivel de significancia de .002 , lo que supone una diferenciación de grupos. La principal variable del instrumento de autoeficacia que distingue a los dos grupos de en función de la depresión es la autoeficacia académica, en tanto la que menos diferencia es la de control (ver Tabla 5), información similar a la obtenida por la prueba $t$.
Tabla 5. Muestra las correlaciones de las variables predictoras con las funciones discriminantes

Correlaciones con

Funciones Discriminantes

\begin{tabular}{ll}
\hline puntaje total de Autoeficacia $^{a}$ & .876 \\
subescala Autoeficacia social & .785 \\
subescala Autoeficacia de control & .308 \\
\hline
\end{tabular}




\section{Autoeficacia y Rendimiento Académico}

La relación entre el rendimiento académico de cada una de las materias y la autoeficacia total y sus tres factores fue analizada a través de correlaciones las cuales resultaron todas ser moderadas, positivas y significativas $(p<.001)$. En términos generales, el promedio general de calificaciones $\mathrm{y}$ de las diferentes asignaturas correlacionan en menor grado con la autoeficacia de control pues presentan índices de correlación que van de .271 a 410; en tanto que los índices con la autoeficacia académica son mayores pues están en un rango que va de .470 a .674 . Con respecto a esta última, existe una correlación moderadamente alta con el desempeño en seis asignaturas, Educación Estatal $(r=.674)$ Ciencias Sociales $(r=.599)$, Matemáticas $(r=.594)$, Inglés $(r=.591)$, Español $(r=.550)$ y Tecnológica $(r=.535)$.

\section{Autoeficacia y Dinámica Familiar}

Para identificar las relaciones entre la autoeficacia y la dinámica familiar se realizaron diversas correlaciones. Los puntajes totales de los instrumentos de autoeficacia y del clima familiar, muestran una correlación muy baja y no significativa $(r=.183, p=.105)$. No obstante atendiendo a los componentes de cada instrumento se advierte que las relaciones de cohesión y conflicto en la familia correlacionaron moderadamente bajo y significativamente tanto con el total de la escala de autoeficacia, así como con las diversas subescalas que la componen, aunque hay que señalar que en el caso del conflicto la correlación es negativa (ver Tabla 6). Otra subescala del clima familiar que correlaciona de manera baja pero significativamente con la autoeficacia es la que valora el aspecto del desarrollo moral y religioso. Por otra parte hay que resaltar que la autoeficacia de control correlacionó sólo con las relaciones de conflicto de manera negativa.

Tabla 6. Muestra las correlaciones entre los diversos puntajes de la escala de Autoeficacia y de la Escala de Clima Social en la Familia.

\begin{tabular}{|c|c|c|c|c|c|}
\hline \multicolumn{2}{|c|}{ Escala de Clima Social en la Familia } & \multicolumn{4}{|c|}{ Escala de Autoeficacia } \\
\hline Dimensiones & Escalas & Total & Académica & Social & Control \\
\hline \multirow{3}{*}{ Relaciones } & Cohesión & $.316(* *)$ & $.288(* *)$ & $.325(* *)$ & .160 \\
\hline & Expresividad & .216 & .163 & $.225(*)$ & .191 \\
\hline & Conflicto & $-.437(* *)$ & $-.374(* *)$ & $-.428(* *)$ & $-.309(* *)$ \\
\hline \multirow[t]{5}{*}{ Desarrollo } & Autonomía & .054 & .022 & .041 & .115 \\
\hline & Actuación & .092 & .132 & .068 & -.039 \\
\hline & Intelectual-Cultural & .134 & .098 & .154 & .104 \\
\hline & Social-Recreativo & -.047 & -.072 & .030 & -.050 \\
\hline & Moral-Religioso & $.292(* *)$ & $.250(*)$ & $.292(* *)$ & .197 \\
\hline \multirow[t]{2}{*}{ Estabilidad } & Organización & .183 & $.249(*)$ & .057 & .055 \\
\hline & Control & -.052 & .046 & -.153 & -.133 \\
\hline
\end{tabular}

$* p<.05 ; * * p<.001$

Con la intención de detectar como se manifestaban estas relaciones entre los estudiantes deprimidos severos y no deprimidos se realizó un análisis de correlación para cada uno de esos dos grupos, cuyos resultados pueden verse en la Tabla 7. En ella se nota que la cohesión familiar correlaciona positivamente con la autoeficacia total y con el factor de autoeficacia académica en los sujetos sin depresión. La expre- sividad en la familia influye positivamente en el factor de autoeficacia de control y en la autoeficacia total. Son otras las relaciones que se manifiestan en los sujetos deprimidos severos y sus familias, en ellos se advierte que las relaciones familiares conflictivas correlacionan de manera negativa tanto en el factor de autoeficacia social como en la autoeficacia total.

Tabla 7. Muestra al interno de cada grupo, sujetos sin depresión y sujetos deprimidos severos, las correlaciones entre los diversos puntajes de la escala de Autoeficacia y de la Escala de Clima Social en la Familia.

\begin{tabular}{|c|c|c|c|c|c|c|c|c|}
\hline \multirow[t]{2}{*}{ Clima Social en la Familia } & \multicolumn{4}{|c|}{ Sujetos sin depresión } & \multicolumn{4}{|c|}{ Sujetos con depresión severa } \\
\hline & $\begin{array}{l}\text { autoefica- } \\
\text { cia total }\end{array}$ & $\begin{array}{l}\text { autoeficacia } \\
\text { académica }\end{array}$ & $\begin{array}{l}\text { autoeficacia } \\
\text { social }\end{array}$ & $\begin{array}{l}\text { autoeficacia de } \\
\text { control }\end{array}$ & $\begin{array}{l}\text { autoeficacia } \\
\text { total }\end{array}$ & $\begin{array}{l}\text { autoeficacia } \\
\text { académica }\end{array}$ & $\begin{array}{l}\text { autoeficacia } \\
\text { social }\end{array}$ & $\begin{array}{l}\text { autoeficacia } \\
\text { de control }\end{array}$ \\
\hline Total & 0.253 & 0.251 & 0.129 & 0.249 & -0.203 & -0.179 & -0.104 & -0.221 \\
\hline Relaciones Cohesión & $.379\left(^{*}\right)$ & $.355\left(^{*}\right)$ & 0.29 & 0.311 & -0.023 & -0.066 & 0.113 & -0.073 \\
\hline Relaciones Expresividad & $.349(*)$ & 0.289 & 0.225 & $.413(* *)$ & -0.149 & -0.202 & 0.036 & -0.137 \\
\hline Relaciones Conflicto & -0.255 & -0.168 & -0.234 & $-.319(*)$ & $-.357(*)$ & -0.27 & $-.381(*)$ & -0.242 \\
\hline Desarrollo Social - Recreativo & -0.065 & -0.072 & -0.097 & 0.021 & $-.313(*)$ & $-.363\left(^{*}\right)$ & -0.083 & -0.231 \\
\hline $\begin{array}{l}\text { Desarrollo Moralidad - Reli- } \\
\text { giosidad }\end{array}$ & 0.17 & 0.177 & 0.108 & 0.124 & $.321(*)$ & 0.21 & $.388(*)$ & 0.239 \\
\hline
\end{tabular}




\section{Discusión}

De acuerdo a Bandura, Barbaranelli, Caprara y Pastorelli (1996), un elevado nivel de autoeficacia percibida aumenta la motivación y el logro académico, lo cual también ocurre en la muestra estudiada en este trabajo dado que el rendimiento académico se relaciona positivamente con la autoeficacia académica.

La depresión es un factor que interactúa de manera importante en la autoeficacia. En este trabajo la depresión se asoció negativamente con la autoeficacia académica en un nivel moderado bajo de manera también significativa, relación similar a la encontrada en otras investigaciones (Bandura, Pastorelli, Barbaranelli y Caprara, 1999; Bandura et al., 1996; Carrasco y Del Barrio, 2002), es decir los resultados presentes son consonantes con la literatura: una alta percepción de eficacia se relaciona con bajas puntuaciones en depresión. Una baja autoeficacia percibida se relaciona con la sintomatología depresiva, mostrando en los individuos sesgos en el procesamiento cognitivo, incumplimiento de aspiraciones, falta de percepción de control e ineficacia social. (Bandura et al., 1999).

La forma en que la depresión se relaciona con la autoeficacia pareciera ser similar, en algunos aspectos, a la manera en que la ansiedad se relaciona con la autoeficacia. Existen reportes que muestran que la ansiedad y la autoeficacia se relacionan de manera negativa, baja y significativa (Zimmerman, 1999 y Contreras et al, 2005). Las creencias de autoeficacia ejercen influencia positiva en la percepción de los individuos sobre su capacidad para desempeñar las tareas requeridas y en las reacciones emocionales favorables ya que no sólo refuerzan el nivel de logro sino que proporcionan bienestar. Los procesos afectivos, como la depresión, ansiedad y el nivel de activación del arousal, son influidos por las creencias de autoeficacia. En este caso las escasas percepciones de autoeficacia conducen a dudar de las propias capacidades y generar sentimientos negativos, una sensación de ansiedad, de falta de control, de desamparo y /o de depresión.

Esto concuerda con los datos presentes en la literatura; en el caso de adolescentes, se ha encontrado que aquellos quienes creen que no pueden controlar las demandas escolares y establecer y mantener relaciones satisfactorias con sus iguales, sufren frecuentes síntomas depresivos (Bandura et al.,1996). Pastorelli, Barbaranelli, Bandura y Caprara (1996, citados en Bandura 1997) y Carrasco y Del Barrio (2002) hallan, similarmente a los datos presentados, relaciones entre la sintomatología depresiva y la ineficacia percibida en los dominios académico, social y de autocontrol en la vida de los adolescentes.

Comparando otros aspectos, la ansiedad y la depresión difieren en su relación con el rendimiento académico y la autoeficacia académica. En otros reportes la ansiedad se encuentra asociada sólo con la autoeficacia y no con el rendimiento académico (Zimmerman, 1999 y Contreras et al, 2005), en tanto que en este estudio la depresión se relacionó tanto con la autoeficacia como con el rendimiento académi- co. Particularmente, de acuerdo al análisis discriminante efectuado, es la autoeficacia académica la que distingue a los sujetos con depresión severa y sin ella.

Las posibles razones de esta diferencia pueden deberse a que se tienen evidencias de que la ansiedad moderada puede favorecer el aprendizaje de algunas asignaturas y entorpecer el de otras (Polaino, 1993), de tal suerte que en el desempeño global escolar esta influencia quede neutralizada y por ello no se encuentren relaciones estadísticamente significativas entre ansiedad y rendimiento académico. Con respecto a la depresión, la relación con el rendimiento académico es negativa y se presenta tanto en las puntuaciones globales del desempeño académico como en las puntuaciones de las diversas asignaturas cursadas por los estudiantes, y aún cuando para algunas asignaturas tal relación pudiera ser significativa y para otras no, en todos los casos la relación es negativa (Campo-Arias et al, 2005; Galicia et al, 2007; Pérez y Urquijo, 2001). Dado que en los adolescentes su desempeño escolar se encuentra asociado a la aprobación social, se convierte en un área muy importante en sus vidas. Un bajo rendimiento académico se relaciona con bajas posibilidades de aceptación social, lo cual puede conducir a una baja autoeficacia percibida en la cual se enfaticen los desilusiones y por ello aumenten los cuadros depresivos

El tener en este estudio a los participantes divididos en función de su nivel de depresión, posibilita observar de manera más precisa la relación entre rendimiento académico, autoeficacia académica y depresión. Estos tres factores, se encuentran íntimamente interrelacionados, al respecto se puede suponer que los sujetos identificados con depresión severa tienen un rendimiento académico bajo porque están influenciados por un bajo sentido de eficacia, ésto a su vez pudiera estar causado por dos aspectos, uno de ellos relacionado con el procesamiento sesgado de las experiencias, desmereciendo o distorsionando negativamente los logros y realzando los fracasos, de forma que los sujetos atribuyen dichos fracasos a sí mismos (Alden, 1986) y el otro aspecto se relacionaría con un bajo sentido de eficacia social para desarrollar relaciones satisfactorias, que aminoren los efectos de estresores crónicos. Con respecto a este último aspecto se puede mencionar que el soporte social reduce la vulnerabilidad a las enfermedades físicas, el estrés y la depresión. Dado que el soporte social no es una entidad que espera ser usada, las personas deben buscar crear relaciones de apoyo por ellas mismas. Esto último requiere un alto sentido de eficacia social. Por lo tanto, un bajo sentido de eficacia para crear relaciones satisfactorias, que den apoyo a la persona, favorece la depresión directa e indirectamente constriñe la evolución de soportes sociales (Bandura, 1997 y Ruiz, 2005). Esta consideración se ve apoyada con los datos obtenidos en los que se advierte que los adolescentes con depresión severa muestran niveles de autoeficacia académica y social más bajos que los adolescentes sin depresión.

Si bien se ha mencionado que la autoeficacia es la percepción de logro hacia alguna actividad particular y no es generalizable a otras, hay quienes sostienen que se puede 
hablar de una autoeficacia general. Algunos autores consideran la autoeficacia en un sentido amplio, entendiendo esta autoeficacia general como un constructo global que hace referencia a la creencia estable que tiene un individuo sobre su capacidad para manejar adecuadamente una amplia gama de estresores de la vida cotidiana (Sanjuán, Pérez-García y Bermúdez, 2000). La autoeficacia general refleja una generalización de unos tipos de autoeficacia a diferentes dominios de funcionamiento dando como resultado que las personas también se valoren como autoeficaces en diversos aspectos. De tal suerte que la autoeficacia general pudiera explicar un amplio rango de comportamientos humanos cuando el contexto es poco específico. En algunos estudios se ha encontrado una relación importante entre una baja autoeficacia general y ansiedad y depresión en estudiantes y en personas enfermas (Luszczynska, Scholz, Schwarzer, 2005). Para argumentar esta posición se puede aludir que aún cuando el individuo aprende patrones autorregulatorios en un campo determinado, puede ocurrir que algunos de esos patrones pudieran ser aplicables a otros campos, de tal suerte que pudiera hablarse no sólo de patrones particulares sino también de procesos autorregulatorios generales. De los resultados generados en este estudio se podría sugerir que la insuficiente autoeficacia académica presentada por los estudiantes identificados con depresión severa podría deberse también a un escaso desarrollo de patrones autorregulatorios y que podrían relacionarse quizás con una escasa autorregulación no sólo de las relaciones sociales, como se advirtió en los datos, sino también con una escasa autorregulación de estados emocionales. Como es sabido, la mayor fuente de estrés no sólo es la frecuencia de pensamientos perturbadores, sino también la falta de habilidad para nulificarlos. Así pues, es posible que los individuos con depresión severa tengan una escasa autorregulación para disminuir dichos pensamientos. Para fundamentar esta suposición habría que realizar un estudio en el que se analice la presencia de patrones autorregulatorios y la posible existencia de una relación entre la autoeficacia académica y la autoeficacia emocional, tanto en estudiantes con y sin depresión.

Por otra parte, para Contreras et al. (2005) el que el rendimiento académico general se encuentre asociado de manera directa con la autoeficacia y no con la ansiedad es un índice de que la ansiedad ejerce un efecto indirecto en el rendimiento académico debido a su relación con la autoeficacia, quien lo ejerce de manera directa. En este caso el rendimiento académico se asocia directamente con la autoeficacia y también con la depresión, lo que conduciría a sugerir que la depresión ejerce un efecto directo en el rendimiento académico. No obstante habrá que reforzar tal suposición no sólo con la evidencia que al respecto proporcionan diversos estudios, sino también realizando una investigación ex profeso para tal objetivo, la cual tendría que comprender no sólo un mayor número de participantes, sino también que estos sean elegidos aleatoriamente para no tener que distinguirlos por alguna característica como la depresión. Se tendrían que aplicar instrumentos para evaluar la depresión y ansiedad en la misma muestra y emplear algún modelo estadístico para encontrar la relación en la que se manifiestan las variables en cuestión

Atendiendo los datos generales obtenidos, en lo que se refiere a la relación entre autoeficacia y dinámica familiar se puede afirmar que las escalas relativas a la dimensión de relaciones del FES son las que principalmente se relacionan con la autoeficacia percibida, tanto en lo general como en cada uno de los factores de la escala empleada. De manera más particular, la evidencia surgida de los datos generales sugiere que posiblemente una manera en que los alumnos analizados conforman una alta autoeficacia social y académica tiene que ver con las relaciones de cohesión establecidas en su núcleo familiar. Se puede decir que la capacidad percibida que tienen los alumnos analizados para dirigir su propio aprendizaje, y lograr las expectativas académicas personales, parentales y de los profesores, así como la capacidad percibida para establecer relaciones entre iguales de tipo asertivo y realizar actividades de ocio y tiempo libre, están influenciadas en la manera como la familia se mantiene unida, en como se ayudan entre sí y se encuentran compenetrados. También se advierte que el nivel de importancia que la familia otorga a los procesos de desarrollo personal, especialmente en lo que se refiere a las prácticas y valores de tipo ético y religioso, es un factor que contribuye a la percepción de autoeficacia académica y social. Este punto resulta interesante pues entre los aspectos no explorados en la autoeficacia que Usher y Pajares (2008) proponen como elementos futuros a indagar en la conformación de la autoeficacia escolar y académica se encuentra precisamente el aspecto religioso. Proponen que la indagación de la pertinencia de este aspecto, así como de otros elementos, constituiría el siguiente paso en la investigación de la autoeficacia; señalan además que deberá hacerse desde una aproximación ecológica para investigar la manera en que tales elementos pueden nutrir la autoeficacia. De ahí que quizás un aspecto a indagar en futuros estudios sea el del papel de los aspectos religiosos en la familia como elementos nutritivos de la autoeficacia en general y en la autoeficacia social y académica.

Retomando la dimensión de Relaciones se puede ver una estrecha relación, pero de carácter negativo entre la escala de conflicto y todos los factores evaluados de autoeficacia. En la medida en que la cólera y la agresividad se expresen libre y abiertamente en la familia, no se favorecen las autopercepciones de los ámbitos académico y social pues éstas son bajas. La frecuente presencia de conflictos entre los miembros de una familia puede influir para que en alguno de sus miembros adolescentes se presente un bajo sentido para desarrollar relaciones satisfactorias no sólo en el ambiente familiar sino también en el escolar y se produzcan bajas expectativas de autoeficacia de tipo académico y social.

Dado que la autoeficacia implica procesos autorregulatorios habría que indagar cómo éstos pudieran estar presentes o favorecidos por la dinámica familiar. Esto podría explorarse a través de tres escalas de la FES, la autonomía, la organización y el control. Con respecto a la primera escala, en ella 
se plasma el grado en que los miembros de la familia están seguros de sí mismos, son autosuficientes y toman sus propias decisiones. Una de las maneras en que la familia favorecería la autonomía sería por medio del establecimiento de comportamientos que logren que sus integrantes, en especial los jóvenes, puedan conocerse así mismos para que de manera progresiva vayan tomando la dirección de su vida, logren la satisfacción de sus necesidades y la consecución de sus metas. Los adolescentes que conformaron la muestra estudiada reportan una baja autonomía en sus familias, y no se encuentran diferencias entre los que tienen depresión y los que no la tienen. Tampoco se encuentran correlaciones entre la autonomía y los tres factores de la autoeficacia percibida. Lo anterior permite plantear que al no existir una relación entre la autonomía y la autoeficacia percibida, podría ser que los procesos autorregulatorios promovidos en la familia no guardan relación con los procesos autorregulatorios de la autoeficacia

Otras escalas que pudieran estar relacionadas con los procesos autorregulatorios son organización y control. Estas escalas constituyen la dimensión de estabilidad, que se refieren a la estructura y organización de la familia y al grado de control que normalmente ejercen unos miembros de la familia sobre otros. Sólo la escala de organización se correlacionó con la autoeficacia académica. Dado que esta escala valora la importancia que la familia le da a la planificación de las actividades y una clara estructura de las mismas, es factible que, aún cuando los patrones de comportamiento ordenado y organizado establecidos en el hogar sean moderados, de alguna manera ayudan al adolescente a tener orden y control en actividades relacionadas con los aspectos escolares y académicos y ello se relacione con la autoeficacia académica percibida.

Los datos mostraron puntajes moderados en la escala de control (que son mayores que los que se presentaron en la escala de organización); lo anterior indica que en buena medida la vida familiar de los dos grupos de sujetos se atiene a reglas y procedimientos establecidos, sin embargo no se encuentran correlaciones entre esos puntajes y los de autoeficacia. Por una parte se puede suponer que las normas impuestas o los procedimientos de control establecidos en las familias de los estudiantes analizados no influyen en la percepción de autocontrol quizás porque las normas son impuestas por los adultos y no se toman en cuenta las propuestas de los adolescentes. Esto puede hacer que las consideren ajenas a ellos y por lo mismo no las estimen importantes para regular su comportamiento. Por otra parte, la falta de relación entre el autocontrol evaluado en la escala de autoeficacia y el control aludido en la escala FES, se debe en parte a que valoran aspectos diferentes. En el primero se valora la capacidad percibida por el sujeto para resistirse a los iguales ante la involucración en actividades de riesgo, relacionadas con la transgresión de normas; en tanto que en la escala FES el control, como ya fue referido, se refiere al grado en que la vida familiar se rige por reglas. En el primero se trata de una autorregulación, ante situaciones más bien de carácter social establecida entre pares, en tanto que en el segundo se trata de una regulación externa. Aquí habría que cuestionarse en que medida la regulación externa incide en una autorregulación. Resulta lógico suponer que para que se desarrolle la autorregulación, primero tendrá que ocurrir una regulación externa. Dado que algunos autores consideran que la adolescencia temprana es una de las fases críticas de la vida para el aprendizaje autorregulatorio (Zimmerman, Bonner y Kovach 1996 y Pajares, 1996), sería interesante indagar, en futuras investigaciones, cuáles son los patrones autorregulatorios ya establecidos en la adolescencia y la manera en que se interrelacionan entre ellos y con los patrones de regulación externa.

$\mathrm{Al}$ averiguar como se manifiestan las relaciones antes expresadas en los dos grupos de individuos analizados se nota que el vínculo entre la dinámica familiar y la autoeficacia se constituye diferencialmente en los individuos que tienen un problema emocional. Mientras en los adolescentes sin depresión la autoeficacia académica se relaciona positivamente con la cohesión familiar, en los adolescentes con depresión severa, la autoeficacia académica establece una relación negativa con el desarrollo social-recreativo. Otro aspecto que destaca es que aún cuando en el desarrollo de la religiosidad no se presentaron diferencias significativas entre los adolescentes con depresión severa y sin ella, la religiosidad ayuda a los adolescentes con depresión severa a percibirse autoeficaces socialmente, en tanto que en los adolescentes sin depresión no se encuentra una relación particular de la autoeficacia social y la dinámica familiar. Es interesante observar que las relaciones conflictivas en la familia son importantes pues son las que distinguen a los dos grupos estudiados, sin embargo influyen en ellos de manera diferente, en los individuos sin depresión se advierte que los conflictos familiares reducen la autoeficacia de control y en los sujetos con depresión severa, reducen la autoeficacia social. Este es un dato interesante pues de acuerdo a Jenkins, Goodnes y Buhrmester (2002) el rol del conflicto en la depresión ha sido muy poco abordado en la adolescencia, algunas evidencias parecen indicar que hay niveles de conflicto que pueden ser adecuados para las relaciones familiares y sociales, en tanto que hay datos contrarios señalando la asociación entre los conflictos familiares y cuadros depresivos en niños y adolescentes de edad temprana, no obstante los estudios no pueden establecer predicciones, amén de que la relación puede ser bidireccional e influida por el género; los conflictos pueden incrementar la depresión, al menos en las chicas, y es factible que la depresión intervenga en los conflictos en el caso de los varones. El estudio actual dirige la atención del papel de la relación entre el conflicto familiar y la depresión hacia las percepciones de eficacia social y de autocontrol.

Estas consideraciones y los hallazgos de este trabajo llevan a estimar que aún cuando se pueden encontrar algunas relaciones generales que muestren la mediación de las relaciones familiares con la autoeficacia y en especial con la autoeficacia académica, éstas pueden diferir en la manera en que inciden en los sujetos dependiendo de diversos factores 
siendo uno de ellos su estado emocional, como se muestra en este estudio en el que se abordó el nivel de depresión de los individuos.

\section{Consideraciones finales}

Los cambios en la adolescencia pueden tener efectos profundos en las creencias acerca de las capacidades del adolescente para triunfar en los ámbitos que se desenvuelve Las instituciones educativas deben ir más allá de la enseñanza de destrezas de trabajo intelectual, y desarrollar en los estudiantes creencias y capacidades autorregulatorias. Se sugiere que se capacite a los estudiantes para observar y autoevaluar su efectividad en el estudio, establecer metas y utilizar estrategias de aprendizaje, así como supervisar y refinar esas estrategias en función de los resultados. Una forma de lograrlo pudiese consistir en motivar a los jóvenes a alcanzar metas que ellos mismos se planteen, y exponerles modelos académicos y sociales positivos, sin olvidar enseñarles estrategias que puedan utilizar para sobreponerse a retos. Así mismo se sugiere que se les oriente para que desarrollen habilidades de regulación emocional, con las cuales los adolescentes se vuelvan eficientes en los intentos que realizan para influenciar sus emociones, en decidir cuándo tenerlas, en experimentarlas y expresarlas adecuadamente así como en modificar sus emociones negativas. Esto con el objeto de que emociones y sentimientos negativos, así como las ideas nocivas, que pudieran estar presentes en los adolescentes no lleguen a manifestarse en cuadros depresivos. Lo anterior debe ser acompañado con talleres destinados a los padres, en los que se les instruya para que promuevan actividades que aumenten la cohesión familiar y reduzcan los conflictos, dado que ese tipo de relaciones son las que se vieron asociadas tanto a la depresión como a la autoeficacia académica y social.

Para los adolescentes es muy importante el apoyo que puedan tener no sólo de los padres sino también de sus pares pues éstos conforman una fuente importante de apoyo social; en la adolescencia temprana se ha encontrado una mayor importancia del apoyo brindado por la familia en tanto que posteriormente son las relaciones y apoyo de sus pares las que los adolescentes más estiman e influyen en ellos (Jenkins, Goodnes y Buhrmester, 2002). En este estudio los participantes se encuentran en la etapa temprana de la adolescencia y se advierte la importancia del apoyo brindado por la familia, pero de manera diferencial. De ahí que se sugiera

\section{Referencias}

Alden, L. (1986). Self-efficacy and causal attributions for social feedback. Journal of Research in Personality, 20, 460-473.

Avendaño, M. J. y Barra, E. (2008). Autoeficacia, apoyo social y calidad de vida en adolescentes con enfermedades crónicas. Terapia Psicológica, 26 (2), 165-172.

Bandura, A. (1997). Self efficacy: the exercise of control. New York: W. H. Freeman. realizar programas que fortalezcan no sólo el apoyo de los padres sino también de otras personas importantes como los compañeros y amigos de los adolescentes, en especial en aquellas escuelas donde sus integrantes se encuentren en la etapa de la adolescencia tardía.

Otra propuesta es ocuparse en fortalecer la autoeficacia filial, es decir fortalecer la eficacia percibida del adolescente para hablar con sus padres sobre sus problemas personales, expresar sentimientos positivos y manejar reacciones emocionales negativas hacia sus padres; a su vez, hacer que sus padres entiendan su punto de vista en diversos temas, manejar el estrés que surge por problemas maritales, e influenciar a sus padres actitudes y prácticas sociales de manera constructiva, incluso cuando las situaciones son tensas (Caprara, Pastorelli, Regalia, Scabini y Bandura 2005). Trabajos como los de Gómez, Barreal y Guillén (2009), muestran que los talleres de autoeficacia filial incrementan especialmente la comunicación de los padres hacia los hijos y viceversa, además de reducir moderadamente las relaciones de hostilidad y rechazo, aspectos de la dinámica familiar que, de acuerdo a los análisis realizados en este trabajo, inciden de manera importante en la depresión, la autoeficacia académica y social y el rendimiento académico.

Caprara et al. (2005) sugieren que mientras los adolescentes se encuentren más convencidos de su capacidad para manejar sus relaciones con sus padres, más probable será que confíen en ellos respecto a sus preocupaciones, actividades y los dilemas que enfrentan en sus experiencias sociales fuera de casa. Desde esta perspectiva, se espera que la autoeficacia filial juegue un papel fundamental en cuanto a propiciar comunicación abierta entre los adolescentes y sus padres, en prevenir el agravamiento de conflictos, en propiciar un monitoreo adecuado, promover patrones autorregulatorios en diferentes esferas, entre ellas la académica, y, por último en encaminar a los adolescentes hacia una vida adulta satisfactoria. De acuerdo a los planteamientos y hallazgos empíricos de la línea de investigación de la autoeficacia, el bienestar humano se relaciona estrechamente con las creencias de autoeficacia. Según esta perspectiva, las personas consiguen su bienestar como consecuencia del logro de sus objetivos personales, los cuales dependen de una manera importante de las creencias de autoeficacia. Esta relación entre la autoeficacia y el bienestar se ha revelado como un factor importante en el mantenimiento del bienestar a través de los diferentes ciclos vitales.

Bandura, A., Barbaranelli, C., Caprara, G. y Pastorelli, C. (1996). Multifaceted impact of self-efficacy beliefs on academic functioning. Child development, 67, 1206-1222.

Bandura, A., Pastorelli, C., Barbaranelli, C. y Caprara, G.V. (1999). Selfefficacy pathways to childhood depression. Journal of Personality and Social Psychology, 76 (2), 258-269. 
Barrera, F. y Vargas, E. (2005). Relaciones familiares y cogniciones románticas en la adolescencia: el papel mediador de la autoeficacia romántica. Revista de Estudios Sociales, 21, 27-35.

Breso E., Salanova M., Martínez I. M., Grau R. y Agut S. (2004). Éxito académico y expectativas de éxito: el rol mediador de la autoeficacia académica. En M. Salanova (Comp.), Nuevos Horizontes en la Investigación sobre Autoeficacia, (pp. 237-342). Castellón (España): Universitat Jaume I.

Campo-Arias, A., González, S., Sánchez, Z., Rodríguez, D., Dallos, C. y Díaz-Martínez, L. (2005). Percepción de rendimiento académico y síntomas depresivos en estudiantes de media vocacional de Bucaramanga, Colombia. Archivos Pediátricos Uruguayos, 76 (1), 21-26. Recuperado el 3 de octubre de 2010 de: http://www.scielo.edu.uy/scielo.php? script $=$ sci_arttext\&pid $=$ S0004-05842005000100005\&lng $=$ es\&nrm $=$ iso.

Caprara, G.V., Pastorelli, C., Regalia, C., Scabini, E. y Bandura A.(2005). Impact of adolescents' filial self-efficacy on quality of family functioning and satisfaction. Journal of Research on Adolescence, 15(1), 71-97.

Carrasco, M. A. y Del Barrio, M. V. (2002). Evaluación de la autoeficacia en niños y adolescentes. Psicothema, 14 (2), 323-332.

Contreras, F., Espinosa, J., Esguerra, G., Haikal, A., Polanía, A. y Rodríguez, A. (2005). Autoeficacia, ansiedad y rendimiento académico en adolescentes. Diversitas, 1 (2), 183-194.

De la Peña, F., Estrada, A., Almeida, L. y Páez, F. (1999). Prevalencia de los trastornos depresivos y su relación con el bajo aprovechamiento escolar es estudiantes de secundaria. Salud Mental, 22 (4), 9-13.

Ellias, S. y Ross, J. (2002). Utilizing Need for Cognition and Perceived SelfEfficacy to Predict Academic Performance. Journal of Applied Social Psychology, 32 (8), 1687-1702.

Galicia, I., Sánchez, A. y Robles, F.J. (2009). Factores asociados a la depresión en adolescentes: rendmiento escolar y dinámica familiar. Anales de Psicología, 25 (2), 227-240.

Gómez, J., Barreal, M. y Guillen, N. (2009). Aplicación de un programa de autoeficacia filial para optimizar la relación filial en adolescentes. Ajayu, 7 (2), 193-212.

Jenkins, S., Goodnes, K. y Burhmester, D. (2002). Gender differences in early adolescents' relationship qualities, self-efficacy, and depression symptoms. Journal of Early Adolescence, 22, (3), 277-309.

Luszczynska, A., Scholz, U. y Schwarzer, R. (2005). The general self-efficacy scale: Multicultural validation studies. The Journal of Psychology, 139 (5), 439-457.

Pajares, F. (1996). Self-efficacy beliefs in academic settings. Review of Educational Research, 66, 543-578.

Pastorelli, C., Caprara, G.V., Barbaranelli, C., Rola, J., Rozsa, S. y Bandura, A. (2001). The structure of children's perceived self-efficacy: A crossnational study. European Journal of Psychological Assessment, 17, 87-97.

Pérez, E. (2006). Inventario de autoeficacia para el estudio: desarrollo y validación inicial. Avaliação Psicológica, 5(2), 35-143.
Pérez G. A., Bermúdez, M. J. y Sanjuán S. P. (2000). Escala de autoeficacia general: datos psicométricos de la adaptación para población española. Psicothema, 12 (2), 509-513.

Pérez, E. y Delgado, M. F. (2006). Inventario de autoeficacia para el estudio: desarrollo y validación inicial. Avaliação psicológica, 5 (2), 135-143.

Pérez, M. y Urquijo, S. (2001). Depresión en adolescentes. Relaciones con el desempeño académico. Psicología Escolar Educativa, 5 (1), 49-58. Recuperado el 3 de octubre de 2007 de: http://pepsic.bvspsi.org.br/scielo.php?script $=$ sci_arttext\&pid $=$ S14138557200100010000 6\&lng $=\mathrm{pt \& n} r \mathrm{~m}=$ iso.

Polaino, A. (1993). Procesos afectivos y aprendizaje: intervención psicopedagógica. En J. Beltrán y cols. (Eds.), Intervención psicopedagógica (pp. 108142). Madrid: Pirámide.

Rey, M., Blasco, T. y Borrás, F. X. (2000). Efectos de un procedimiento de inducción de estados de ánimo sobre la autoeficacia. Anales de psicología 16 (1), 23-31.

Rueda, B. y Pérez-Garcia, A. (2004). Personalidad y percepción de autoeficacia: Influencia sobre el bienestar y el afrontamiento de los problemas de salud. Revista de Psicopatología y Psicología Clínica, 9 (3), 205-219.

Ruiz, F. (2005). Influencia de la autoeficacia en el ámbito académico. Revista Digital de Investigación en Docencia Universitaria, 1 (1). Recuperado el 5 de diciembre de 2010 de: http:/ / beta.upc.edu.pe/calidadeducativa/ridu/ 2007/ridu4_3FL.pdf.

Sanjuán, P., Pérez, A. M. y Bermúdez, J. (2000). Escala de autoeficacia general: datos psicométricos de la adaptación para población española. Psicothema, 12, (2), 509-513.

Schunk, D. y Meece, J. (2005). Self-efficacy development in adolescences. En F. Pajares y T. Urdan (Eds.), Self-efficacy believes during adolescence. Greenwich, C.T.: Informations Age Publishing.

Schnewind, K. (1999). Impacto de los proesos familiares sobre las creencias de control. En A. Bandura (Ed.), Autoeficacia: Cómo afrontamos los cambios de la sociedad actual. Bilbao: Desclée de Brouwer.

Seisdedos, N., De la Cruz, M. V. y Cordero, A. (1995). Escalas de Clima Social. Madrid: TEA

Usher, E. y Pajares, F. (2008). Sources of self efficacy in school: critical review of the literature and future directions. Review of Educational Research. 78, (4), 752-796.

Zimmerman, B. (1999). Autoeficacia y desarrollo educativo. En: A. Bandura (Ed.), Autoeficacia: Cómo afrontamos los cambios de la sociedad actual. Bilbao: Desclée de Brower.

Zimmerman, B., Bonner, S. y Kovach, R. (1996). Developing self-regulated learners. Beyond achievement to self-efficacy. Washington: American Psychological Association.

Zimmerman, B. J., Kitsantas, A. y Campillo, M. (2005). Evaluación de la Autoeficacia Regulatoria: Una Perspectiva Social Cognitiva. Evaluar, 5, 1 21.

(Artículo recibido: 3-4-2011, revisado: 02-04-2012, aceptado: 02-05-2012) 